\title{
Bi-oriented Quantum Algebras, and a Generalized Alexander Polynomial for Virtual Links
}

\author{
Louis H. Kauffman and David Radford
}

\begin{abstract}
This paper discusses the construction of a generalized Alexander polynomial for virtual knots and links, and the reformulation of this invariant as a quantum link invariant. We then introduce the concept of a bi-oriented quantum algebra, which provides an algebraic context for this structure.
\end{abstract}

\section{Introduction}

In this paper we discuss the construction of a generalized Alexander polynomial $G_{K}(s, t)$ via the concept of a biquandle. Our approach leads directly to a generalization of the Burau representation upon which this invariant is based. We then reformulate the invariant as a quantum link invariant and as a state summation. In this context we show that the normalized quantum invariant $Z_{K}(\sigma, \tau)$ satisfies a Conway skein identity and reproduces $G_{K}(s, t)$. These invariants are useful for the theory of virtual knots and links, for they vanish on classical knots and links. Hence the invariants studied here can be used to show that many virtual knots and links are not classical. We give such examples in Section 2. We also give an example of a virtual knot that cannot be detected by the generalized Alexander polynomial. This knot is detected by the structure of the corresponding generalized Alexander module. We conclude with a diagram due to Kishino that is knotted, but is not detected by any representation of the biquandle at the time of this writing. We conjecture that the biquandle of the Kishino diagram does detect its knottedness.

In the final section of the paper, we formulate the concept of a bi-oriented quantum algebra. This generalizes our previous concept of oriented quantum algebra $[6,7]$ to include the necessary structures to create invariants of virtual links. The invariant $Z_{K}(\sigma, \tau)$ studied in this paper fits non-trivially into this framework. Subsequent papers will study the structure and applications of bi-oriented quantum algebras.

1991 Mathematics Subject Classification. 57M25.

Key words and phrases. virtual knot, Alexander polynomial, state summation, biquandle, bioriented quantum algebra. 
In relation to the theme of diagrammatics for this proceedings, we should mention that the crossings and R-matrices for the quantum knot invariant models are, respectively, topological and algebraic realizations of the categorical concept of braiding. Similarly, virtual crossings and the operator $\gamma$ of section 5 realize symmetries (which are braidings satisfying Condition 1 of the definition of a bioriented quantum algebra in section 5), and the cups and caps, and $M_{a b}$ of section 4 realize the rigidities of duality. All these devices arise naturally in the context of the generalization of quantum link invariants to virtual knot theory.

\section{Virtual Links and a Generalized Alexander Polynomial}

In this section we will construct a generalization of the Alexander module and Alexander polynomial that is defined for virtual knots and links $[\mathbf{9}, \mathbf{1 0}, \mathbf{1 1}]$. We then show how this generalized Alexander polynomial can be seen as state summation model using a solution to the Quantum Yang-Baxter equation. This state model will form the basis for the rest of the paper.

Recall that classical knot theory can be described in terms of knot and link diagrams. A diagram is a 4-regular plane graph (with extra structure at its nodes representing the crossings in the link) represented on the surface of a plane and implicitly on the surface of a two-dimensional sphere $S^{2}$. One says that two such diagrams are equivalent if there is a sequence of moves of the types indicated in part (A) of Figure 1 (The Reidemeister Moves) taking one diagram to the other. These moves are performed locally on the 4-regular plane graph (with extra structure) that constitutes the link diagram.

Virtual knot theory is an extension of classical knot theory. In this extension one adds a virtual crossing (See Figure 1) that is neither an over-crossing nor an under-crossing. We shall refer to the usual diagrammatic crossings, that is, those without circles, as real crossings to distinguish them from the virtual crossings. A virtual crossing is represented by two crossing arcs with a small circle placed around the crossing point.

The allowed moves on virtual diagrams are a generalization of the Reidemeister moves for classical knot and link diagrams. We show the classical Reidemeister moves as part (A) of Figure 1. These classical moves are part of virtual equivalence where no changes are made to the virtual crossings. Taken by themselves, the virtual crossings behave as diagrammatic permutations. Specifically, we have the flat Reidemeister moves (B) for virtual crossings as shown in Figure 1. In Figure 1 we also illustrate a basic move $(\mathrm{C})$ that interrelates real and virtual crossings. In this move an arc going through a consecutive sequence of two virtual crossings can be moved across a single real crossing. In fact, it is consequence of moves (B) and $(\mathrm{C})$ for virtual crossings that an arc going through any consecutive sequence of virtual crossings can be moved anywhere in the diagram keeping the endpoints fixed and writing the places where the moved arc now crosses the diagram as new virtual crossings. This is shown schematically in Figure 1.1. We call the move in Figure 1.1 the detour, and note that the detour move is equivalent to having all the moves of type (B) and (C) of Figure 1. This extended move set (Reidemeister moves plus the detour move or the equivalent moves (B) and (C)) constitutes the move set for virtual knots and links. 
BI-ORIENTED QUANTUM ALGEBRAS, AND A GENERALIZED ALEXANDER POLYNOMIAL FOR VIRTUAL LINKS

There is a useful topological interpretation for this virtual theory in terms of embeddings of links in thickened surfaces. See $[\mathbf{9 ,} \mathbf{1 1}]$. Regard each virtual crossing as a shorthand for a detour of one of the arcs in the crossing through a 1-handle that has been attached to the 2-sphere of the original diagram. The two choices for the 1-handle detour are homeomorphic to each other (as abstract surfaces with boundary a circle) since there is no a priori difference between the meridian and the longitude of a torus. By interpreting each virtual crossing in this way, we obtain an embedding of a collection of circles into a thickened surface $S_{g} \times R$ where $g$ is the number of virtual crossings in the original diagram $L, S_{g}$ is a compact oriented surface of genus $g$ and $R$ denotes the real line. Thus to each virtual diagram $L$ we obtain an embedding $s(L) \longrightarrow S_{g(L)} \times R$ where $g(L)$ is the number of virtual crossings of $L$ and $s(L)$ is a disjoint union of circles. We say that two such surface embeddings are stably equivalent if one can be obtained from another by isotopy in the thickened surfaces, homeomorphisms of the surfaces and the addition or subtraction of empty handles. Then we have the

Theorem $[\mathbf{9}, \mathbf{1 2}]$. If two virtual link diagrams are equivalent then their correspondent surface embeddings are stably equivalent. Any surface embedding is stably equivalent to the image of a virtual diagram.

In [9] this result is sketched. A complete proof will appear in [12]. The surface embedding interpretation of virtuals is useful since it maps their equivalence to a topological question. However, the stabilization makes classification difficult since one cannot rely on any single surface embedding. The diagrammatic version of virtuals embodies the stabilization in the detour moves. We do not know at this writing if the mapping from virtual knots and links to stabilized suface embedddings is injective. The diagrammatic approach to virtual knot theory provides a setting that must be taken seriously in its own right.

It is a fact that two classical knots that are equivalent in the virtual category are equivalent classically [9]. Thus classical knot theory embeds in virtual knot theory. We say that a virtual knot is trivial if it is equivalent to the classical unknotted circle. We say that a virtual knot or link is classical it is equivalent to a classical knot or link. Certainly a virtual knot can be classical without being trivial. 


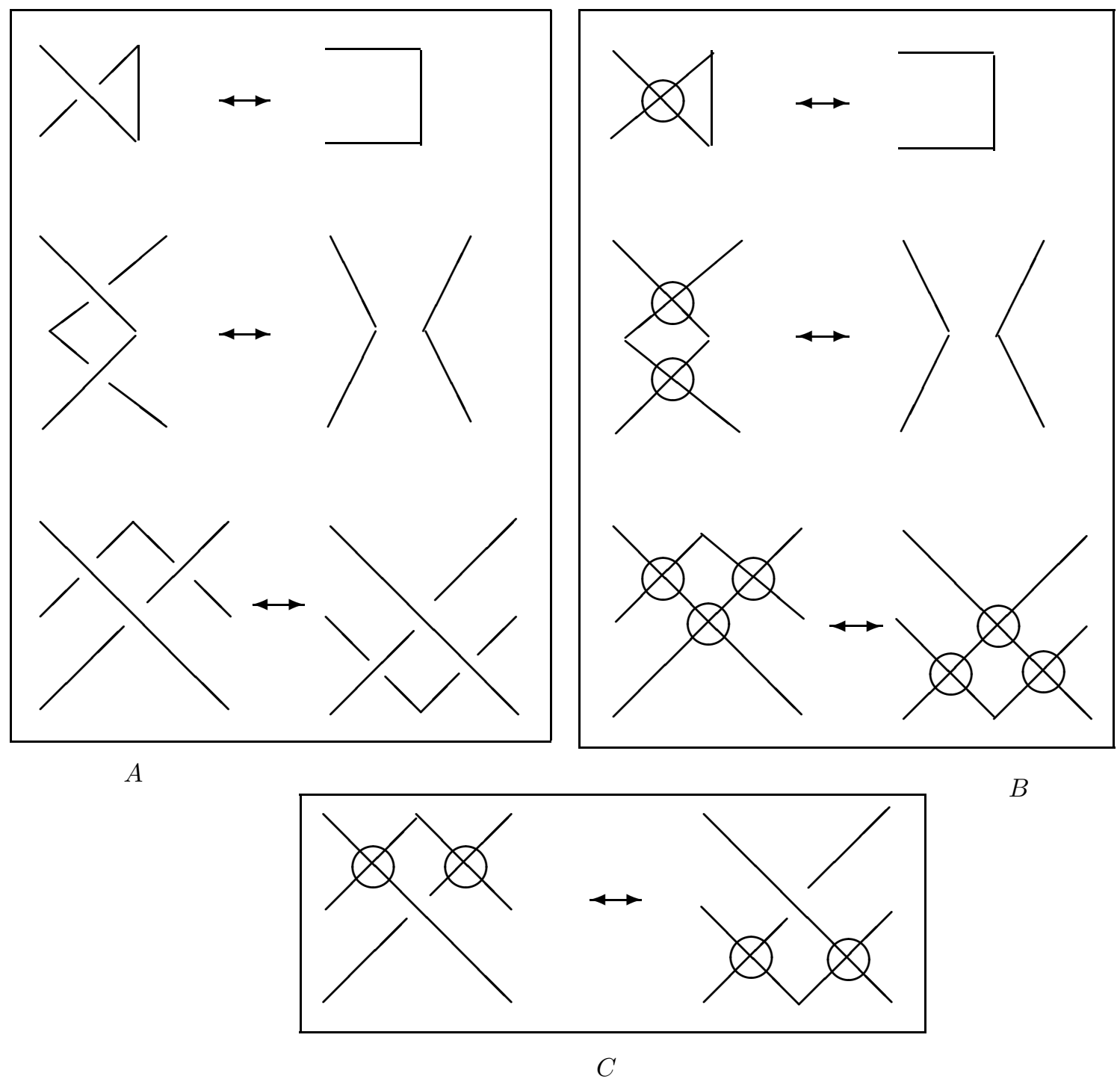

Figure 1 - Generalized Reidemeister Moves for Virtuals 
BI-ORIENTED QUANTUM ALGEBRAS, AND A GENERALIZED ALEXANDER POLYNOMIAL FOR VIRTUAL LINKS
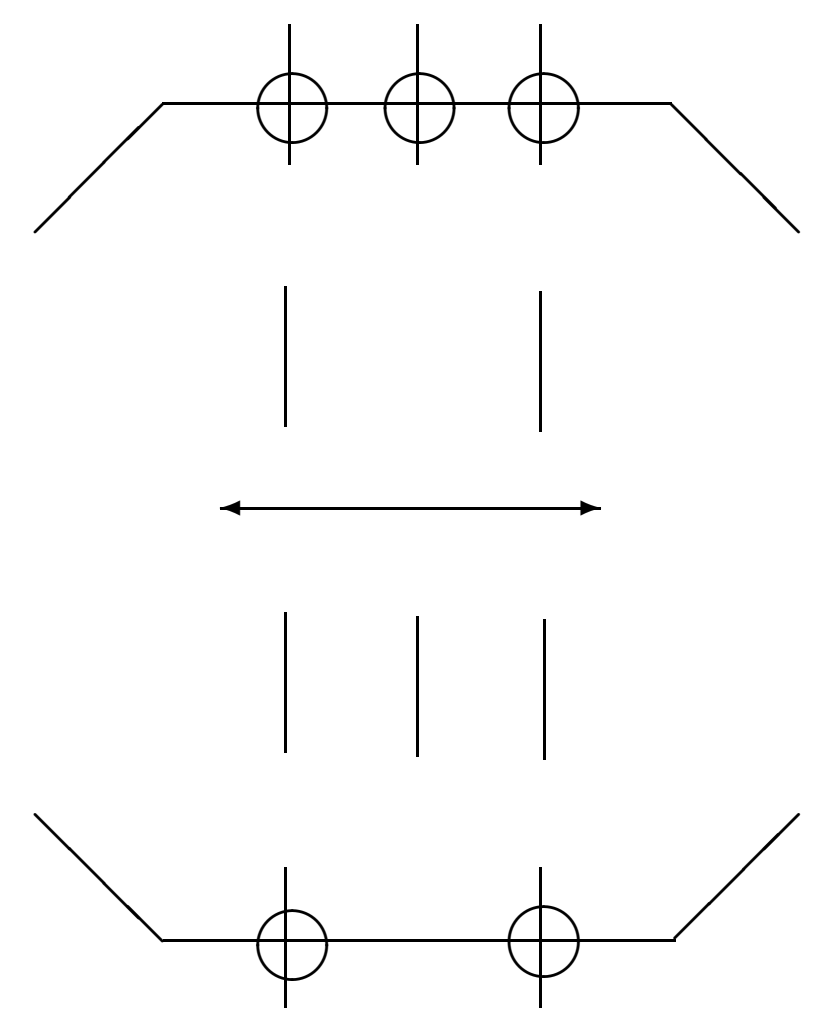

Figure 1.1 - Schema for the Detour Move

\section{Biquandles and a Generalized Alexander Polynomial $G_{K}(s, t)$}

The biquandle $[\mathbf{1 1}, \mathbf{1}, \mathbf{1 6}]$ is an algebraic structure associated with a virtual link diagram that is invariant (up to isomorphism) under the generalized Reidemeister moves for virtual knots and links. We shall refer to the biquandle structure as an algebra. This is meant to denote an algebraic structure satisfying the axioms delineated below. The biquandle is not generically a linear algebra described over some ring, but examples can take this structure. The operations in the biquandle algebra are motivated by the formation of labels for the edges of the diagram and the intended invariance under the moves. We will give the abstract definition of the biquandle after a discussion of these knot theoretic issues. View Figure 2. In this Figure we have shown the format for the operations in a biquandle. The overcrossing arc has two labels, one on each side of the crossing.

Each classical crossing in a virtual link diagram $K$ is regarded as extra structure on a vertex for a 4-regular graph (four edges incident to each vertex) $G(K)$ obtained by projecting the diagram to the plane (if you think of each classical crossing as rising above the plane along one arc). In speaking of graphs we allow a multiplicity of edges between two nodes of the graph, and we allow edges with single endpoints (loops). Each virtual crossing is regarded as the intersection of two edges of the graph $G(K)$ in the planar immersion of that graph that is obtained by projection. The virtual crossings are not nodes of the associated graph. In this way we obtain an immersed 4-regular graph $G(K)$ associated with each virtual link diagram $K$. 
We define an edge of $K$ to be an edge of $G(K)$ lifted to the diagram $K$. That is, an edge of $K$ extends along arcs of the diagram $K$ from one classical crossing to another. In a virtual diagram $D$ with no classical crossings the graph $G(D)$ is a collection of immersed circles in the plane, whose intersections are the virtual crossings of the diagram $D$.

In a biquandle there is an algebra element labeling each edge of the diagram. As described above, an edge of the diagram corresponds to an edge of the underlying graph of that diagram. Let the edges oriented toward a crossing in a diagram be called the input edges for the crossing, and the edges oriented away from the crossing be called the output edges for the crossing. Let $a$ and $b$ be the input edges for a positive crossing (illustrated in the left half of Figure 2), with $a$ the label of the undercrossing input and $b$ the label on the overcrossing input. Then in the biquandle, we label the undercrossing output by

$$
c=a^{b}
$$

just as in the case of the quandle, but the overcrossing output is labeled

$$
d=b_{a} .
$$

We usually read $a^{b}$ as "the undercrossing line $a$ is acted upon by the overcrossing line $b$ to produce the output $c=a^{b}$." In the same way, we can read $b_{a}$ as "the overcossing line $b$ is operated on by the undercrossing line $a$ to produce the output $d=b_{a}$ ". The biquandle labels for a negative crossing (illustrated in the right half of Figure 2) are similar but with an overline (denoting an operation of order two) placed on the letters. Thus in the case of the negative crossing, we would write

$$
c=a^{\bar{b}} \quad \text { and } \quad d=b_{\bar{a}} .
$$

To form the biquandle, $B Q(K)$, we take one generator for each edge of the diagram and two relations at each crossing (as described above). This system of generators and relations is then regarded as encoding an algebra that is generated solely by the biquandle operations as concatenations of these symbols and subject to the biquandle algebra axioms. These axioms (which we will describe below) are a transcription in the biquandle language of the requirement that this algebra be invariant under Reidemeister moves on the diagram.

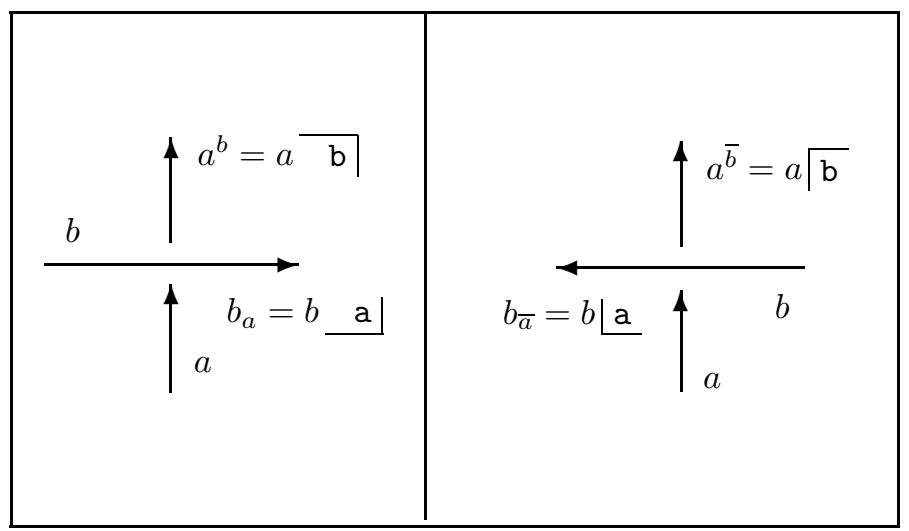

Figure 2 - Biquandle Relations at a Crossing 
Another way to write this formalism for the biquandle is as follows

$$
\begin{aligned}
& a^{b}=a \overline{\mathrm{b}} \\
& a_{b}=a \overline{\mathrm{b}} \\
& a^{\bar{b}}=a \overline{\mathrm{b}} \\
& a_{\bar{b}}=a \underline{\mathrm{b}} .
\end{aligned}
$$

We call this the operator formalism for the biquandle. The operator formalism has advantages when one is performing calculations, since it it possible to maintain the formulas on a line rather than extending them up and down the page as in the exponential notation. On the other hand the exponential notation has intuitive familiarity and is good for displaying certain results. The axioms for the biquandle, are exactly the rules needed for invariance of this structure under the Reidemeister moves. Note that in analyzing invariance under Reidemeister moves, we visualize representative parts of link diagrams with biquandle labels on their edges. The labels throughout each diagrammatic arc will be identified later, providing the equations that the biquandle has to satisfy by definition. Ultimately, the link components will provide, through the structure of the arcs in the diagram, a set of generators for the biquandle, just as cells in $\mathrm{CW}$ complexes become the generators of celluar homology. The primary labeling occurs at a crossing. At a positive crossing with over input $b$ and under input $a$, the under output is labeled $a \overline{\mathrm{b}}$ and the over output is labeled $b$ a $\mid$. At a negative crossing with over input $b$ and under input $a$, the under output is labeled $a \overline{\mathrm{b}}$ and the over output is labeled $b \mid \mathrm{a}$. At a virtual crossing there is no change in the labeling of the lines that cross one another.

Remark. A remark is in order about the relationship of the operator notations with the usual conventions for binary algebraic operations. Let $a * b=a^{b}=a \overline{\mathrm{b}}$. We are asserting that the biquandle comes equipped with four binary operations of which one is $a * b$. Here is how these notations are related to the usual parenthesizations:

$$
\begin{aligned}
& \text { 1. }(a * b) * c=\left(a^{b}\right)^{c}=a^{b c}=a \overline{\mathrm{b}} \overline{\mathrm{c}} \\
& \text { 2. } a *(b * c)=a^{b^{c}}=a \overline{\mathrm{b}} \overline{\mathrm{c}}
\end{aligned}
$$

From this the reader should see that the exponential and operator notations allow us to express biquandle equation with a minimum of parentheses.

In Figure 3 we illustrate the effect of these conventions and how it leads to the following algebraic transcription of the directly oriented second Reidemeister move:

$$
\begin{aligned}
& a=a \overline{\mathrm{b}} \overline{\mathrm{b} \quad \mathrm{a} \mid} \quad \text { or } \quad a=a^{b \overline{b_{a}}} \text {, } \\
& b=b \quad \mathrm{a} \| \mathrm{a} \overline{\mathrm{b}} \quad \text { or } \quad b=b_{a \overline{a^{b}}} .
\end{aligned}
$$

Note that reversing both arrows in Figure 3 has the effect on the equations of reversing all operations right to left:

$$
\begin{aligned}
& a = a \longdiv { \mathrm { b } } \quad \mathrm { b } \mid \mathrm{a} \\
& b=b \mid \underline { \mathrm { a } } \quad \mathrm { a } \longdiv { \mathrm { b } } .
\end{aligned}
$$


The reverse oriented second Reidemeister move gives a different sort of identity, as shown in Figure 4. For the reverse oriented move, we must assert that given elements $a$ and $b$ in the biquandle, then there exists an element $x$ such that

$$
x=a \overline{\mathrm{b}\lfloor\mathrm{x}} \mid, \quad a=x \overline{\mathrm{b}} \text { and } \quad b=b \underline{\mathrm{x}} \underline{\mathrm{a} \mid} .
$$

By reversing the arrows in Figure 4 we obtain a second statement for invariance under the type two move, saying the same thing with the operations reversed: Given elements $a$ and $b$ in the biquandle, then there exists an element $x$ such that

$$
x = a \longdiv { \mathrm { b } \_ \mathrm { x } \rfloor } \quad, \quad a=x \overline{\mathrm{b}} \text { and } b=b \underline{\mathrm{x} \|} \underline{\mathrm{a}} .
$$

There is no neccessary relation between the $x$ in the first statement and the $x$ in the second statement.

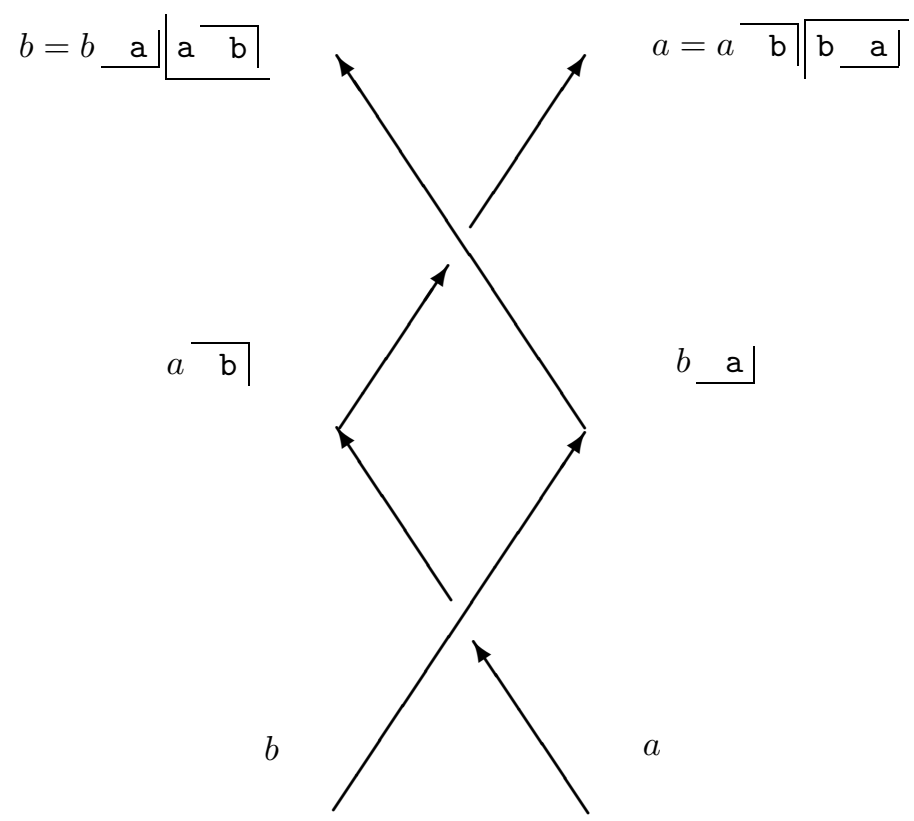

Figure 3 - Direct Two Move 
BI-ORIENTED QUANTUM ALGEBRAS, AND A GENERALIZED ALEXANDER POLYNOMIAL FOR VIRTUAL LINKS

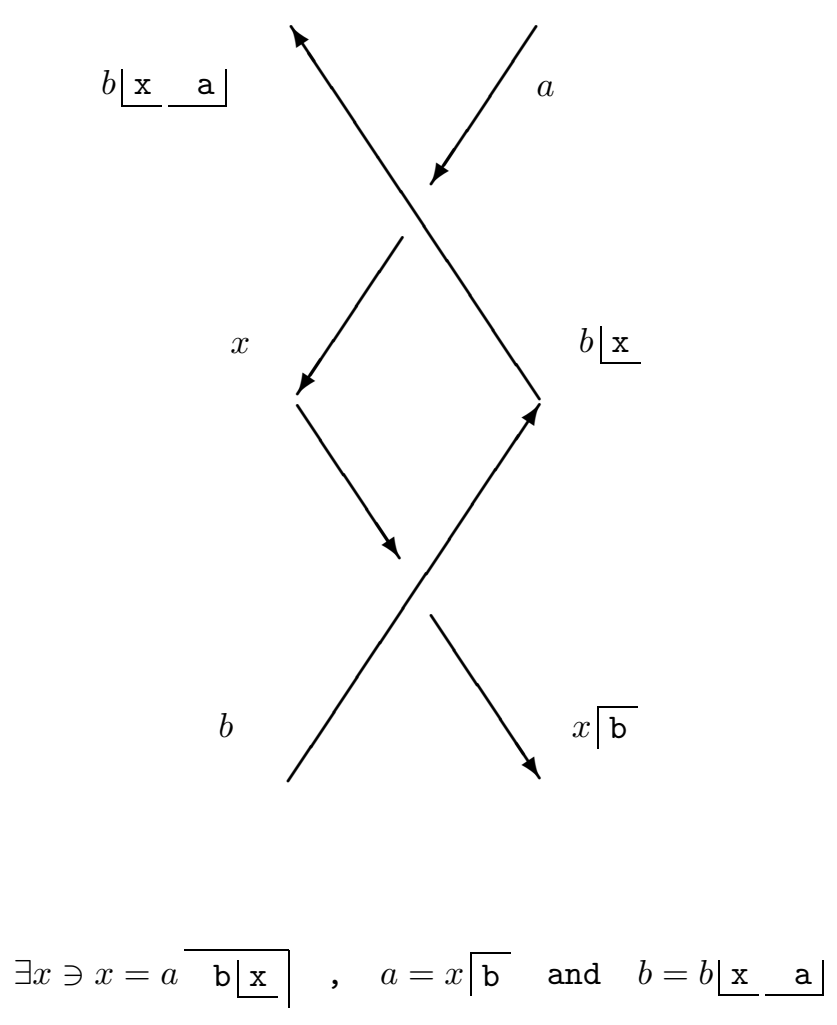

Figure 4 - Reverse Two Move

These assertions about the existence of $x$ can be viewed as asserting the existence of fixed points for a certain operators. In this case such an operator is $F(x)=$ $a \overline{\mathrm{b} \mid \mathrm{x}}$. It is characteristic of certain axioms in the biquandle that they demand the existence of such fixed points. Another example is the axiom corresponding to the first Reidemeister move (one of them) as illustrated in Figure 5. This axiom states that given an element $a$ in the biquandle, then there exists an $x$ in the biquandle such that $x=a_{\mathrm{x}}$ and that $a=x \overline{\mathrm{a}}$. In this case the operator is $G(x)=a \mathrm{x}$. 


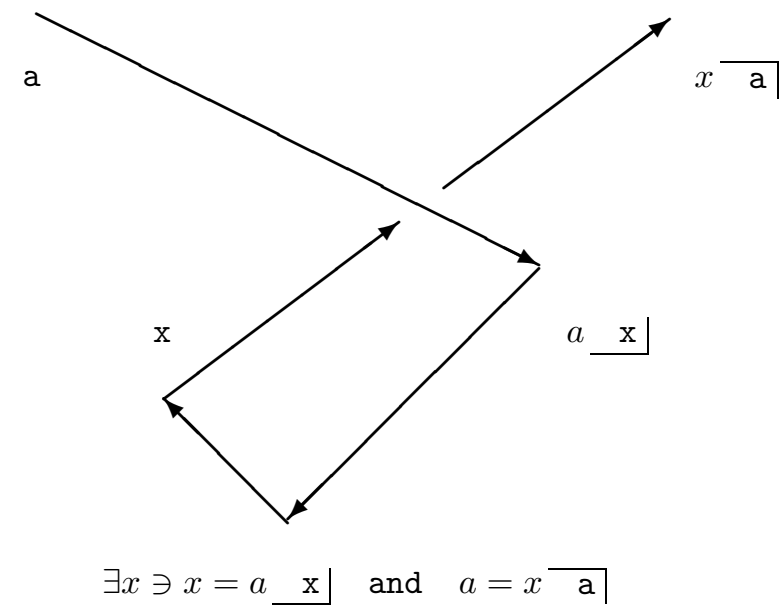

Figure 5 - First Move

It is unusual that an algebra would have axioms asserting the existence of fixed points with respect to operations involving its own elements. We plan to take up the study of this aspect of biquandles in a separate publication.

The biquandle relations for invariance under the third Reidemeister move are shown in Figure 6. The version of the third Reidemeister move shown in this figure yields the algebraic relations:

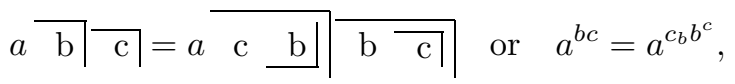

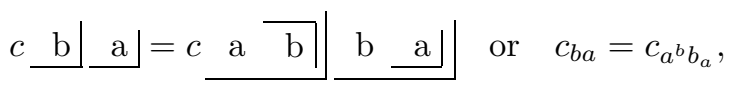

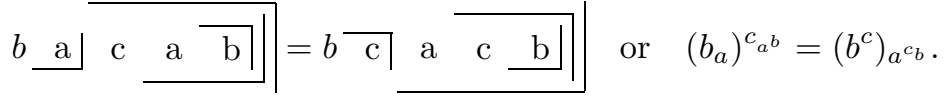


BI-ORIENTED QUANTUM ALGEBRAS, AND A GENERALIZED ALEXANDER POLYNOMIAL FOR VIRTUAL LINK\$
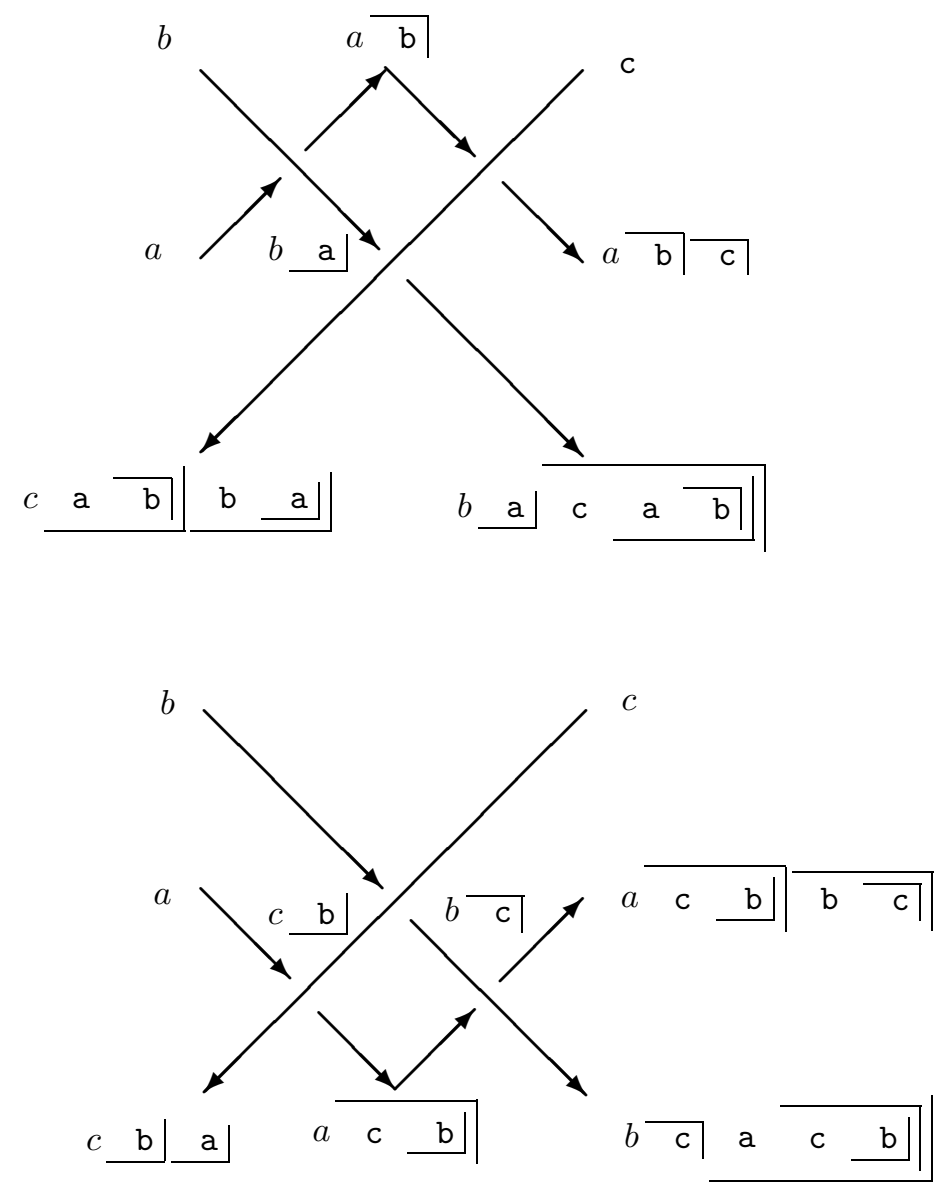

Figure 6 - Third Move

The reader will note that if we replace the diagrams of Figure 6 with diagrams with all negative crossings then we will get a second triple of equations identical to the above equations but with all right operator symbols replaced by the corresponding left operator symbols (equivalently - with all exponent literals replaced by their barred versions). Here are the operator versions of these equations. We refrain from writing the exponential versions because of the prolixity of barred variables.

$$
\begin{aligned}
& a \longdiv { \mathrm { b } } \sqrt { \mathrm { c } } = a \longdiv { \mathrm { c } \lfloor \mathrm { b } } \sqrt { \mathrm { b } \sqrt { \mathrm { c } } },
\end{aligned}
$$

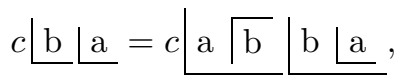

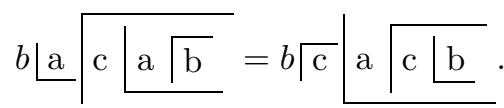

We now have a complete set of axioms, for it is a fact [5] that the third Reidemeister move with the orientation shown in Figure 6 and either all positive crossings (as shown in that Figure) or all negative crossings, is sufficient to generate all the other cases of third Reidemeister move just so long as we have both oriented forms 
of the second Reidemeister move. Consequently, we can now give the full definition of the biquandle.

Definition. A biquandle $B$ is a set with four binary operations indicated by the conventions we have explained above: $a^{b}, a^{\bar{b}}, a_{b}, a_{\bar{b}}$. We shall refer to the operations with barred variables as the left operations and the operations without barred variables as the right operations. The biquandle is closed under these operations and the following axioms are satisfied:

1. For any elements $a$ and $b$ in $B$ we have

$$
\begin{gathered}
a=a^{b \overline{b_{a}}} \quad \text { and } \quad b=b_{a \overline{a^{b}}} \quad \text { and } \\
a=a^{\bar{b} b_{\bar{a}}} \quad \text { and } \quad b=b_{\bar{a} a^{\bar{b}}} .
\end{gathered}
$$

2. Given elements $a$ and $b$ in $B$, then there exists an element $x$ such that

$$
x=a^{b_{\bar{x}}}, \quad a=x^{\bar{b}} \quad \text { and } \quad b=b_{\bar{x} a} .
$$

Given elements $a$ and $b$ in $B$, then there exists an element $x$ such that

$$
x=a^{\overline{b_{x}}}, \quad a=x^{b} \quad \text { and } \quad b=b_{x \bar{a}} .
$$

3. For any $a, b, c$ in $B$ the following equations hold and the same equations hold when all right operations are replaced in these equations by left operations.

$$
a^{b c}=a^{c_{b} b^{c}}, \quad c_{b a}=c_{a^{b} b_{a}}, \quad\left(b_{a}\right)^{c_{a} b}=\left(b^{c}\right)_{a^{c_{b}}} .
$$

4. Given an element $a$ in $B$, then there exists an $x$ in the biquandle such that $x=a_{x}$ and $a=x^{a}$. Given an element $a$ in $B$, then there exists an $x$ in the biquandle such that $x=a^{\bar{x}}$ and $a=x_{\bar{a}}$.

These axioms are transcriptions of the Reidemeister moves. The first axiom transcribes the directly oriented second Reidemeister move. The second axiom transcribes the reverse oriented Reidemeister move. The third axiom transcribes the third Reidemeister move as we have described it in Figure 6. The fourth axiom transcribes the first Reidemeister move. Much more work is needed in exploring these algebras and their applications to knot theory.

3.1. The Alexander Biquandle. In order to realize a specific example of a biquandle structure, suppose that

$$
\begin{aligned}
& a \overline{\mathrm{b}}=t a+v b \\
& a \mathrm{~b}=s a+u b
\end{aligned}
$$

where $a, b, c$ are elements of a module $M$ over a commutative ring $R$ (recall that the additive group structure of a module is given to be commutative) and $t, s, v$ and $u$ are in $R$. We use invariance under the Reidemeister moves to determine relations among these coefficients.

Taking the equation for the third Reidemeister move discussed above, we have

$$
a \overline{\mathrm{b}} \mid \overline{\mathrm{c}}=t(t a+v b)+v c=t^{2} a+t v b+v c
$$


BI-ORIENTED QUANTUM ALGEBRAS, AND A GENERALIZED ALEXANDER POLYNOMIAL FOR VIRTUAL LINKS

$$
\begin{gathered}
a \overline{\mathrm{c} \quad \mathrm{b} \|}|\overrightarrow{\mathrm{b}} \overline{\mathrm{c} \|}|=t(t a+v(s c+u b))+v(t b+v c) \\
=t^{2} a+t v(u+1) b+v(t s+v) c .
\end{gathered}
$$

From this we see that we have a solution to the equation for the third Reidemeister move if $u=0$ and $v=1-s t$. Assuming that $t$ and $s$ are invertible, it is not hard to see that the following equations not only solve this single Reideimeister move, but they give a biquandle structure, satisfying all the moves.

$$
a \overline{\mathrm{b}}=t a+(1-s t) b, \quad a \underline{\mathrm{b}}=s a
$$

$$
a \overline{\mathrm{b}}=t^{-1} a+\left(1-s^{-1} t^{-1}\right) b, \quad a \mid \mathrm{b}=s^{-1} a
$$

Thus we have a simple generalization of the Alexander quandle and we shall refer to this structure, with the equations given above, as the Alexander Biquandle.

Just as one can define the Alexander Module of a classical knot, we have the Alexander Biquandle of a virtual knot or link, obtained by taking one generator for each edge of the knot diagram and taking the module relations in the above linear form. Let $A B Q(K)$ denote this module structure for an oriented link $K$. That is, $A B Q(K)$ is the module generated by the edges of the diagram, modulo the submodule generated by the relations. This module then has a biquandle structure specified by the operations defined above for an Alexnder Biquandle. We first construct the module and then note that it has a biquandle structure. See Figures 7,8 and 9 for an illustration of the Alexander Biquandle labelings at a crossing. 


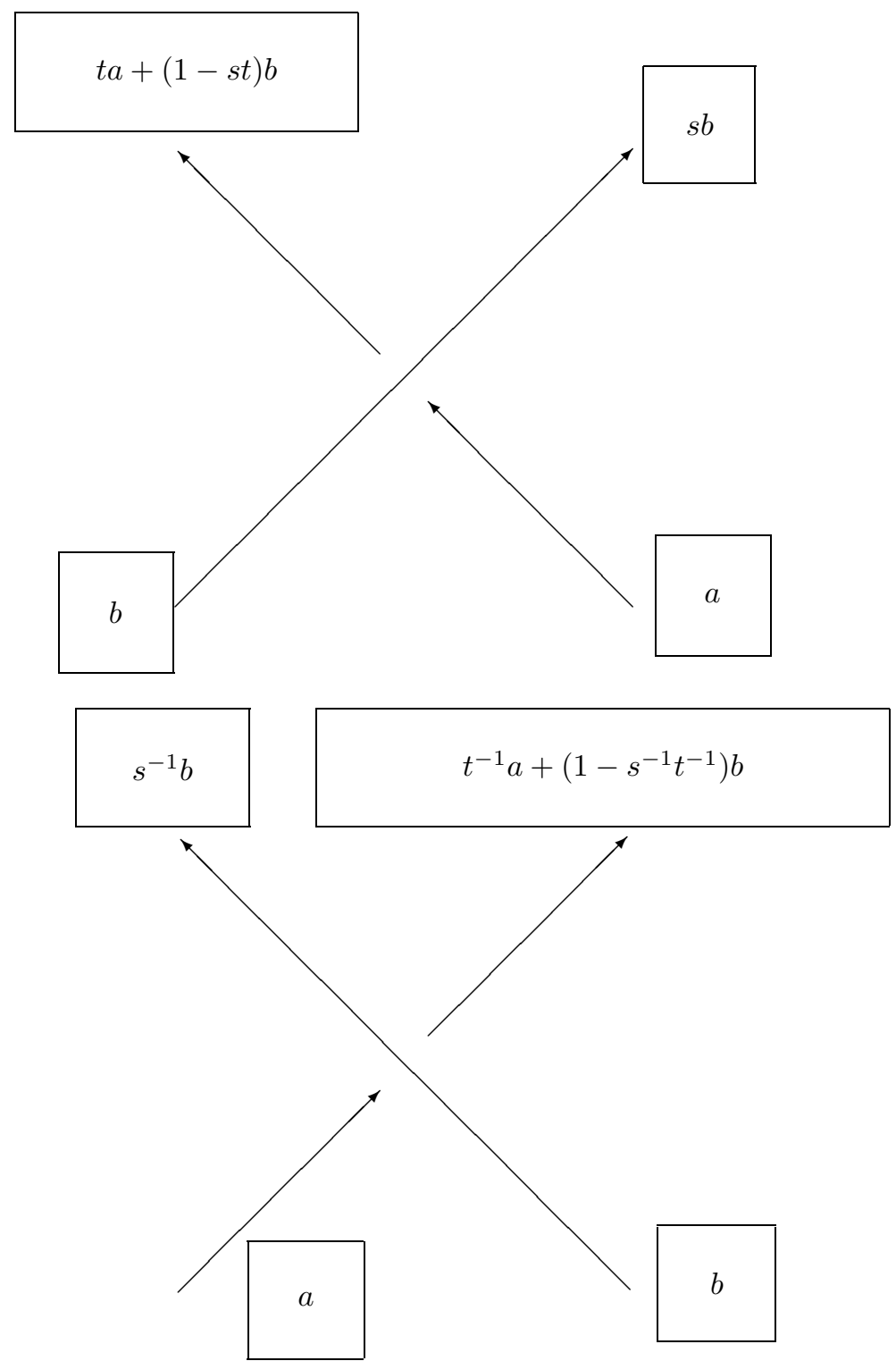

Figure 7 - Alexander Biquandle Labeling at a Crossing 
BI-ORIENTED QUANTUM ALGEBRAS, AND A GENERALIZED ALEXANDER POLYNOMIAL FOR VIRTUAL LINKS

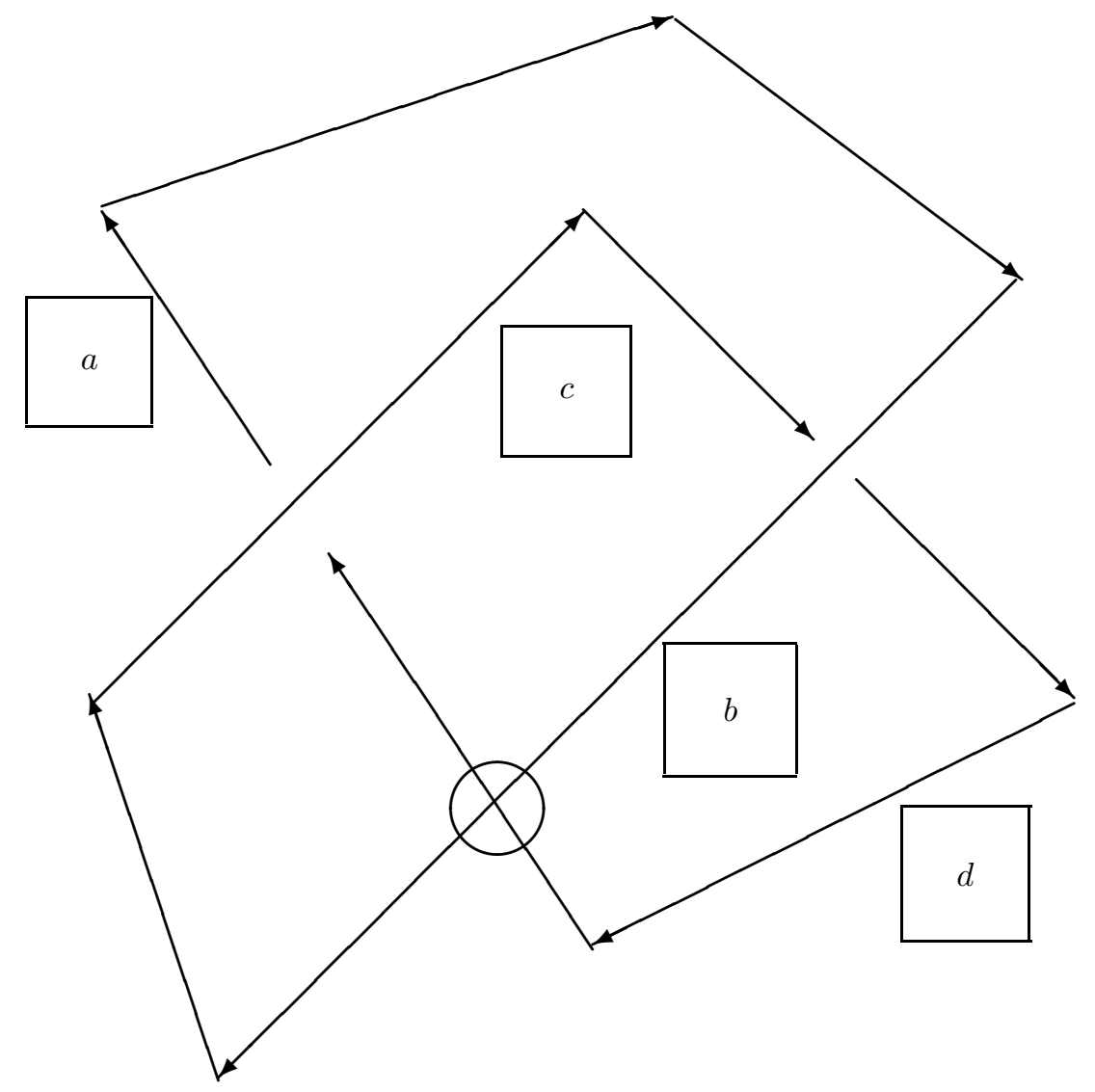

Figure 8 - A Virtual Knot Fully Labeled 


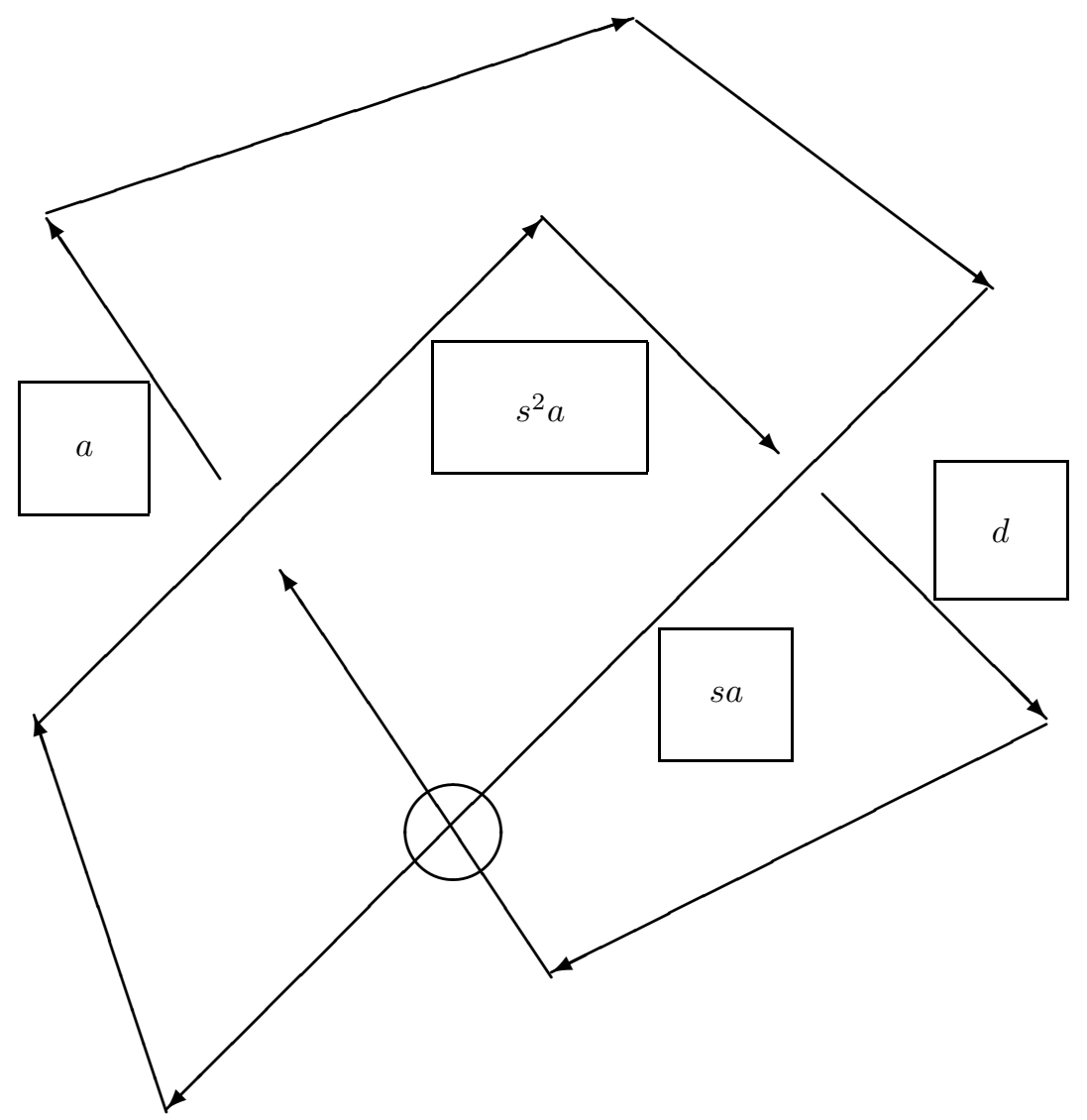

Figure 9 - A Virtual Knot with Lower Operations Labeled

For example, consider the virtual knot in Figure 8. This knot gives rise to a biquandle with generators $a, b, c, d$ and relations

$$
a=d \overline{\mathrm{b}}, \quad c=b \underline{\mathrm{d}}, \quad d=c \overline{\mathrm{a}}, \quad b=a \underline{\mathrm{c}} .
$$

writing these out in $A B Q(K)$, we have

$$
a=t d+(1-s t) b, \quad c=s b, \quad d=t c+(1-s t) a, \quad b=s a .
$$

eliminating $c$ and $b$ and rewriting, we find

$$
\begin{gathered}
a=t d+(1-s t) s a \\
d=t s^{2} a+(1-s t) a
\end{gathered}
$$

Note that these relations can be written directly from the diagram as indicated in Figure 9 if we perform the lower biquandle operations directly on the diagram. This is the most convenient algorithm for producing the relations.

We can write these as a list of relations

$$
\begin{aligned}
& \left(s-s^{2} t-1\right) a+t d=0 \\
& \left(s^{2} t+1-s t\right) a-d=0
\end{aligned}
$$


determining the Alexander Biquandle as a module over $Z\left[s, s^{-1}, t, t^{-1}\right]$. The relations can be expressed concisely with the matrix of coefficients of this system of equations:

$$
M=\left[\begin{array}{cc}
s-s^{2} t-1 & t \\
\left(s^{2} t+1-s t\right) & -1
\end{array}\right]
$$

The determinant of $M$ is, up to multiples of $\pm s^{i} t^{j}$ for integers $i$ and $j$, an invariant of the virtual knot or link $K$. (The elements $\pm s^{i} t^{j}$ are units in the $\operatorname{ring} Z\left[s, s^{-1}, t, t^{-1}\right]$. The relations used for the determinant are determined up to multiplication by units in this ring.) We shall denote this determinant by $G_{K}(s, t)$ and call it the generalized Alexander polynomial for $K$. A key fact about $G_{K}(s, t)$ is that $G_{K}(s, t)=0$ if $K$ is equivalent to a classical diagram. This is seen by noting that in a classical diagram one of the relations will be a consequence of the others.

In this case we have

$$
G_{K}=(1-s)+\left(s^{2}-1\right) t+\left(s-s^{2}\right) t^{2}
$$

which shows that the knot in question is non-trivial and non-classical.

Here is another example of the use of this polynomial. Let $D$ denote the diagram in Figure 10. It is not hard to see that this virtual knot has unit Jones polynomial, and that the combinatorial fundamental group (defined from the diagram by the Wirtinger presentation) is isomorphic to the integers. (A classical knot with the fundamental group of its complement isomorphic to the integers is unknotted by well known results in three dimensional topology. Virtual knots do not have complements, but they do have well-defined combinatorial fundamental groups. See $[\mathbf{9}]$. The biquandle is a generalization of this conbinatorial fundamental group for virtual knots and links.) The biquandle does detect the knottedness of $D$. The relations are

$$
a \overline{\mathrm{d}}=b, d \underline{\mathrm{a}}=e, \quad c \overline{\mathrm{e}}=d, e \underline{\mathrm{c}}=f, \quad f \overline{\mathrm{b}}=a, b\lfloor\mathrm{f}=c
$$

from which we obtain the relations (eliminating $c, e$ and $f$ )

$$
b=t a+(1-t v) d, \quad d=t s^{-1} b+(1-t s) s d, \quad a=t^{-1} s^{2} d+\left(1-t^{-1} s^{-1}\right) b .
$$

The determinant of this system is the generalized Alexander polynomial for $D$ :

$$
t^{2}\left(s^{2}-1\right)+t\left(s^{-1}+1-s-s^{2}\right)+\left(s-s^{2}\right) .
$$

This proves that $D$ is a non-trivial virtual knot. 


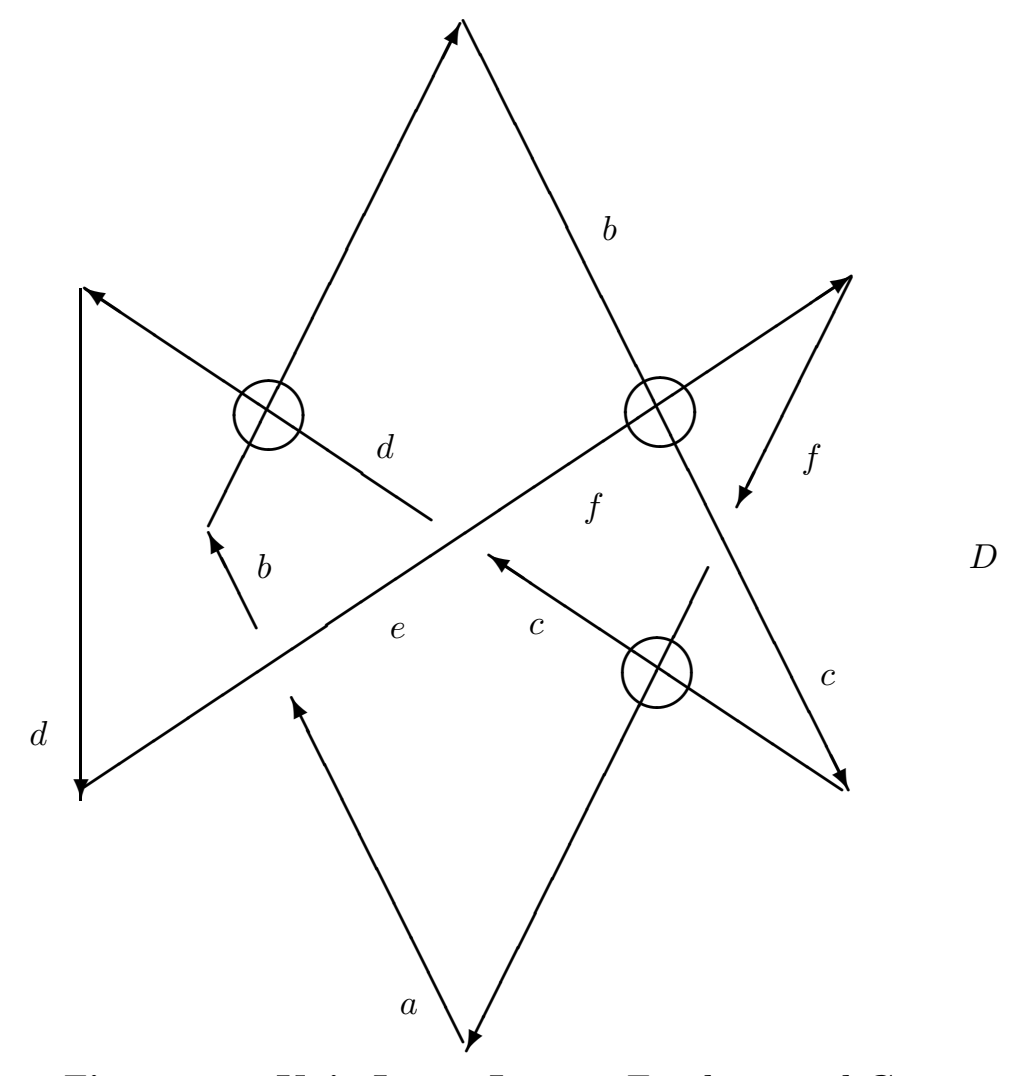

Figure 10 - Unit Jones, Integer Fundamental Group

In fact the polynomial that we have computed is the same as the polynomial invariant of virtuals of Sawollek [14] and defined by an alternative method by Silver and Williams [15]. Sawollek defines a module structure essentially the same as our Alexander Biquandle. Silver and Williams first define a group. The Alexander Biquandle proceeds from taking the abelianization of the Silver-Williams group.

We end this discussion of the Alexander Biquandle with two examples that show clearly its limitations. View Figure 11. In this Figure we illustrate two diagrams labeled $K$ and $K I$. It is not hard to calculate that both $G_{K}(s, t)$ and $G_{K I}(s, t)$ are equal to zero. The Alexander Biquandle of $K$ is non-trivial: Calculation shows that it is isomorphic to the module over $Z\left[s, s^{-1}, t, t^{-1}\right]$ generated by elements $a$ and $b$ subject solely to the relation $\left(s^{-1}-t-1\right)(a-b)=0$. Thus $K$ represents a non-trivial virtual knot. This shows that it is possible for a non-trivial virtual diagram to be a connected sum of two trivial virtual diagrams, and it shows that the Alexander Biquandle can sometimes be more powerful than the polynomial invariant $G$. However, the diagram $K I$ also has trivial Alexander Biquandle and it is not detected by the Silver - Williams group. The diagram $K I$, discovered by Kishino [8] is in fact seen to be a knotted virtual by showhing that it has a non-trivial three strand Jones polynomial [13]. We conjecture that the general biquandle of the Kishino diagram is non-trivial. 
BI-ORIENTED QUANTUM ALGEBRAS, AND A GENERALIZED ALEXANDER POLYNOMIAL FOR VIRTUAL LINKS
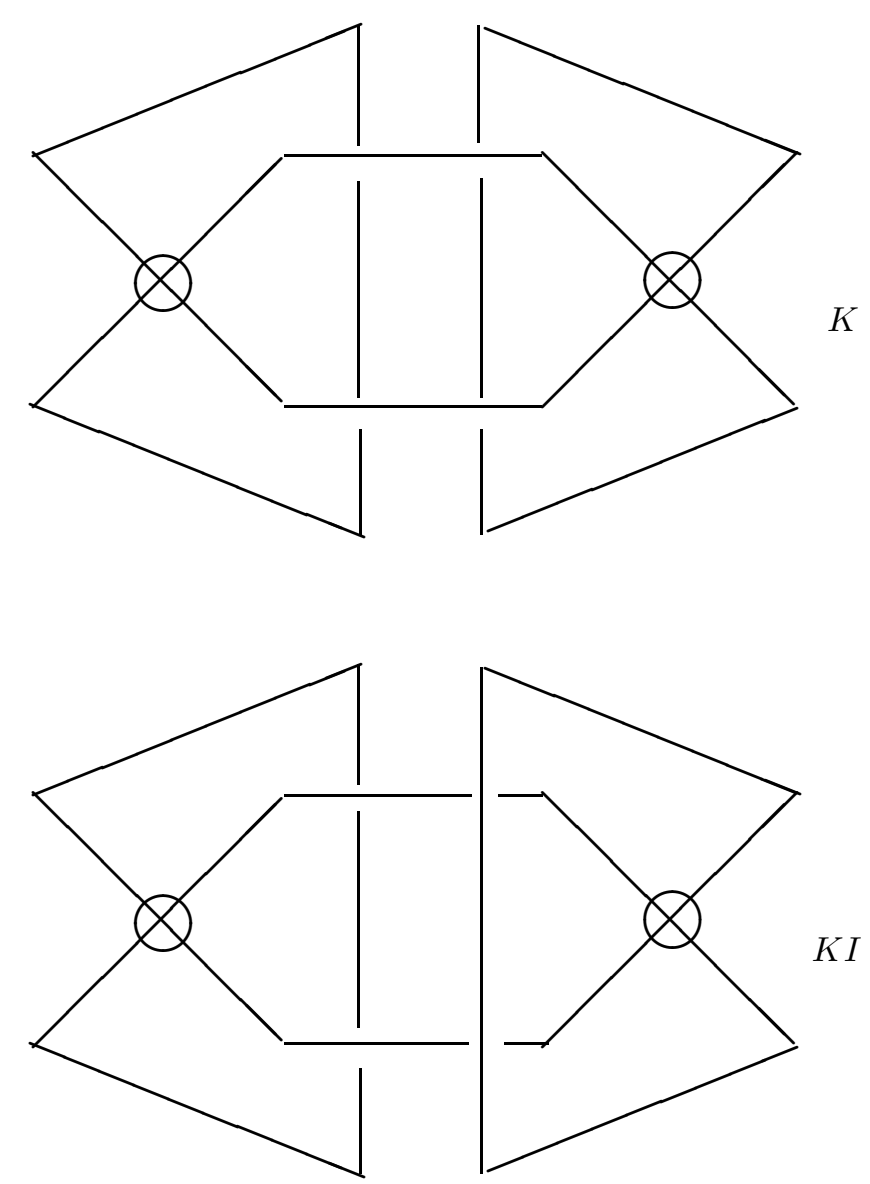

Figure 11 - The Knot $K$ and the Kishino Diagram $K I$

\section{A Quantum Model for $G_{K}(s, t)$}

It is our intent in this paper to analyse the structure of the invariant $G_{K}(s, t)$ by rewriting it as a quantum invariant and then analysing its state summation. We shall show how the quantum invariant and state sum fit into the context of oriented quantum algebras. The quantum model for this invariant is obtained in a fashion analogous to the construction of a quantum model of the Alexander polynomial in $[\mathbf{3}]$ and $[\mathbf{2}]$. The strategy in those papers was to take the basic two dimensional matrix of the Burau representation, view it as a linear transformation $T: V \longrightarrow V$ on a two dimensional module $V$ over a ring of Laurent polynomials in one variable with integer coefficients, and them take the induced linear transformation $\hat{T}: \Lambda^{*} V \longrightarrow \Lambda^{*} V$ on the exterior algebra of $V$. This gives a transformation on a four dimensional module that is a solution to the Yang-Baxter equation. This solution of the Yang-Baxter equation then becomes the building block for the corresponding quantum invariant. In the present instance, we have a generalization of the Burau representation, and this same procedure can be applied to it.

The generalized Burau matrix is given by the formula 
with the inverse matrix

$$
B=\left[\begin{array}{cc}
1-s t & s \\
t & 0
\end{array}\right]
$$

$$
B^{-1}=\left[\begin{array}{cc}
0 & t^{-1} \\
s^{-1} & 1-s^{-1} t^{-1}
\end{array}\right]
$$

The formulas for $B$ and $B^{-1}$ are easily seen by reference to Figure 7 .

We may regard $B$ as acting on a module $V$ with basis $\left\{e_{1}, e_{2}\right\}$ over $Z\left[s, s^{-1}, t, t^{-1}\right]$ via the equations

$$
\begin{gathered}
B e_{1}=(1-s t) e_{1}+t e_{2}, \\
B e_{2}=s e_{1} .
\end{gathered}
$$

Letting $\hat{B}$ denote the extension of $B$ to the exterior algebra on $V$, we have

$$
\begin{gathered}
\hat{B} 1=1, \\
\hat{B} e_{1}=(1-s t) e_{1}+t e_{2}, \\
\hat{B} e_{2}=s e_{1}, \\
\hat{B} e_{1} \wedge e_{2}=\operatorname{Det}(B) e_{1} \wedge e_{2}=-s t e_{1} \wedge e_{2} .
\end{gathered}
$$

Let $R$ denote the matrix of $\hat{B}$ with respect to the basis $\left\{1, e_{1}, e_{2}, e_{1} \wedge e_{2}\right\}$ of the exterior algebra $\Lambda^{*} V$. The matrix $R$ is the matrix of the transformation on the exterior algebra that is induced from the generalized Burau matrix.

$$
R=\left[\begin{array}{cccc}
1 & 0 & 0 & 0 \\
0 & 1-s t & s & 0 \\
0 & t & 0 & 0 \\
0 & 0 & 0 & -s t
\end{array}\right]
$$

$R$ is a $4 \times 4$ matrix solution to the Yang-Baxter equation. Its inverse $\bar{R}$ is shown below.

$$
\bar{R}=\left[\begin{array}{cccc}
1 & 0 & 0 & 0 \\
0 & 0 & t^{-1} & 0 \\
0 & s^{-1} & 1-s^{-1} t^{-1} & 0 \\
0 & 0 & 0 & -s^{-1} t^{-1}
\end{array}\right]
$$

In our case, we also need the induced transformation for the virtual crossing. At the level of the generalized Burau representation, the matrix for the virtual crossing is the $2 \times 2$ matrix for a transposition:

$$
\eta=\left[\begin{array}{ll}
0 & 1 \\
1 & 0
\end{array}\right]
$$

The corresponding matrix induced on the exterior algebra is

$$
\hat{\eta}=\left[\begin{array}{cccc}
1 & 0 & 0 & 0 \\
0 & 0 & 1 & 0 \\
0 & 1 & 0 & 0 \\
0 & 0 & 0 & -1
\end{array}\right] .
$$


This matrix $\hat{\eta}$ is a solution to the Yang-Baxter equation whose square is equal to the identity. It is this operator that will correspond to the virtual crossings in our quantum invariant for virtuals.

Now it is convenient to make some changes of variables for this model. We replace $s$ by $\sigma^{2}$ and $t$ by $\tau^{-2}$. We then replace $R$ by $\sigma^{-1} \tau R$ and $\bar{R}$ by $\sigma \tau^{-1} \bar{R}$. The result is the new matrices shown below, where:

$$
\begin{gathered}
z=\sigma^{-1} \tau-\sigma \tau^{-1} \\
R=\left[\begin{array}{cccc}
\sigma^{-1} \tau & 0 & 0 & 0 \\
0 & z & \sigma \tau & 0 \\
0 & \sigma^{-1} \tau^{-1} & 0 & 0 \\
0 & 0 & 0 & -\sigma \tau^{-1}
\end{array}\right] . \\
\bar{R}=\left[\begin{array}{cccc}
\sigma \tau^{-1} & 0 & 0 & 0 \\
0 & 0 & \sigma \tau & 0 \\
0 & \sigma^{-1} \tau^{-1} & -z & 0 \\
0 & 0 & 0 & -\sigma^{-1} \tau
\end{array}\right] .
\end{gathered}
$$

These matrices plus a choice of cup and cap matrices will define the matrix model for this quantum invariant. See [7] for a description of the matrix models. See [5] for a related discussion of a state summation for the Alexander polynomial. Here the cup and cap matrices are given by the formula:

$$
M_{a b}=(\sqrt{i})^{a} \delta_{a b}
$$

where $i^{2}=-1$ and the matrix $M$ is associated with the clockwise-turning cap and with the clockwise-turning cup, while the matrix $M^{-1}$ is associated with the counterclockwise-turning cap and with the counterclockwise-turning cup. Here the matrix indices are -1 and +1 so that an isolated clockwise loop evaluates as $i^{+1}+$ $i^{-1}=0$. (Remember that this invariant vanishes on classical links.) It is easy to verify that this model is invariant under all but the first Reidemeister moves (classical and flat). Let $W(K)$ denote this evaluation. It is then not hard to see that the following normalization $Z(K)$ creates a function on virtual knots that is invariant under all of the (generalized) Reidemeister moves:

$$
Z(K)=\left(\sigma^{-1} \tau i\right)^{\operatorname{rot}(K)-v(K)} i^{v(K)} W(K)
$$

where $\operatorname{rot}(K)$ denotes the sum of the Whitney degrees of the underlying plane curves of $K$ (where $\operatorname{rot}(K)=1$ when $K$ is a simple clockwise circle in the plane), and $v(K)$ denotes the number of virtual crossings in the diagram $K$.

We can formulate this invariant as a state summation by using the formulas in Figure 12 to expand a given diagram into a sum of evaluations of labeled signed loops in the plane. Each loop has only virtual crossings and the rule for expanding the virtual tells us that only the ++ signing receives a minus one as vertex weight. Note the distinction between the vertex weights (the matrix entries) and the signs (the matrix indices). In all other cases the virtual crossing contributes one as a vertex weight. Once there is such a labeled collection of signed loops, it can be tested for compatibility. A collection of loops is compatible if there is no contradiction in the signs (each curve is uniquely labeled plus or minus). An incompatible labeling corresponds to zero in the state sum. Then each compatible labeled signed collection is evaluated by taking the product of the vertex weights multiplied by the product 
of the evaluations of the signed loops. The evaluation of a given signed loop $\lambda$ is equal to $i^{\epsilon(\lambda) \operatorname{rot}(\lambda)}$ where $\epsilon(\lambda)$ is the sign of the loop and $\operatorname{rot}(\lambda)$ is the Whitney degree of the loop. Note that because of the presence of the virtual crossings, the Whitney degree of a loop can be any integer. The state summation evaluates the unnormalized invariant $W(K)$.

Both $W(K)$ and $Z(K)$ satisfy a skein relation that is just like that of the Conway polynomial:

$$
\begin{gathered}
W\left(K_{+}\right)-W\left(K_{-}\right)=z W\left(K_{0}\right) \\
Z\left(K_{+}\right)-Z\left(K_{-}\right)=z Z\left(K_{0}\right)
\end{gathered}
$$

where $K_{+}, K_{-}$and $K_{0}$ denote three diagrams that differ at one site, with a positive crossing, a negative crossing and a smoothing respectively. This skein relation, illustrated in Figure 12, is useful in relative computations, but there are infinitely many virtual links whose evaluation cannot be decided by the skein relation alone. The simplest example is the "virtual Hopf link" $H$ of Figure 13. In $H$ there is one crossing and one virtual crossing. Switching this crossing does not simplify the link. In Figure 13 we illustrate the state sum calculation of $W(H)$. The Figure shows the four contributing states. It is clear from the Figure that

$$
W(H)=\tau \sigma^{-1}+\tau^{-1} \sigma-\sigma \tau-\sigma^{-1} \tau^{-1}=\left(\tau-\tau^{-1}\right)\left(\sigma^{-1}-\sigma\right)
$$

This calculation is obtained by evaluating the signed loops according to the description given above. In particular, the case of the single loop with plus sign has a rotation number of zero and a multiplicative vertex weight of minus one contributed from the virtual crossing. In all the other cases the vertex weight of the virtual crossing is plus one. The first two states have rotation number zero, while the second two contribute $i^{-2}=-1$ and $i^{2}=-1$ respectively. This explains the signs in the formula for $W(H)$. To obtain $Z(H)$, we note that $\operatorname{rot}(H)=0$ and that $H$ has a single virtual crossing. So

$$
\begin{gathered}
Z(H)=\left(\sigma^{-1} \tau i\right)^{\operatorname{rot}(H)-v(H)} i^{v(H)} W(H)=\left(\sigma^{-1} \tau i\right)^{0-1} i^{1} W(H) \\
=\sigma \tau^{-1} W(H)=\sigma \tau^{-1}\left(\tau-\tau^{-1}\right)\left(\sigma^{-1}-\sigma\right)=\left(1-\tau^{-2}\right)\left(1-\sigma^{2}\right)=(1-t)(1-s) .
\end{gathered}
$$

It is easy to see that this corresponds to the calculation of $G_{H}(s, t)$ by the determinant function. The state summation $Z(H)$ provides a normalized value for the invariant that can be used for skein calculations.

The basic result behind the correspondence of $G_{K}(s, t)$ and $Z(K)$ is the

Theorem. For a (virtual) link $K$, the invariants $Z(K)(\sigma=\sqrt{s}, \tau=1 / \sqrt{t})$ and $G_{K}(s, t)$ are equal up to a multiple of $\pm s^{n} t^{m}$ for integers $n$ and $m$ (this being the well-definedness criterion for $G$ ).

We omit the proof of this result. The argument is the same as that for the relation between the classical Alexander polynomial and its quantum counterpart. See $[\mathbf{3}]$ and $[\mathbf{5}]$. 
BI-ORIENTED QUANTUM ALGEBRAS, AND A GENERALIZED ALEXANDER POLYNOMIAL FOR VIRTUAL LINI\&

$$
\begin{aligned}
& \text { K-1 } \\
& +\sigma \tau
\end{aligned}
$$
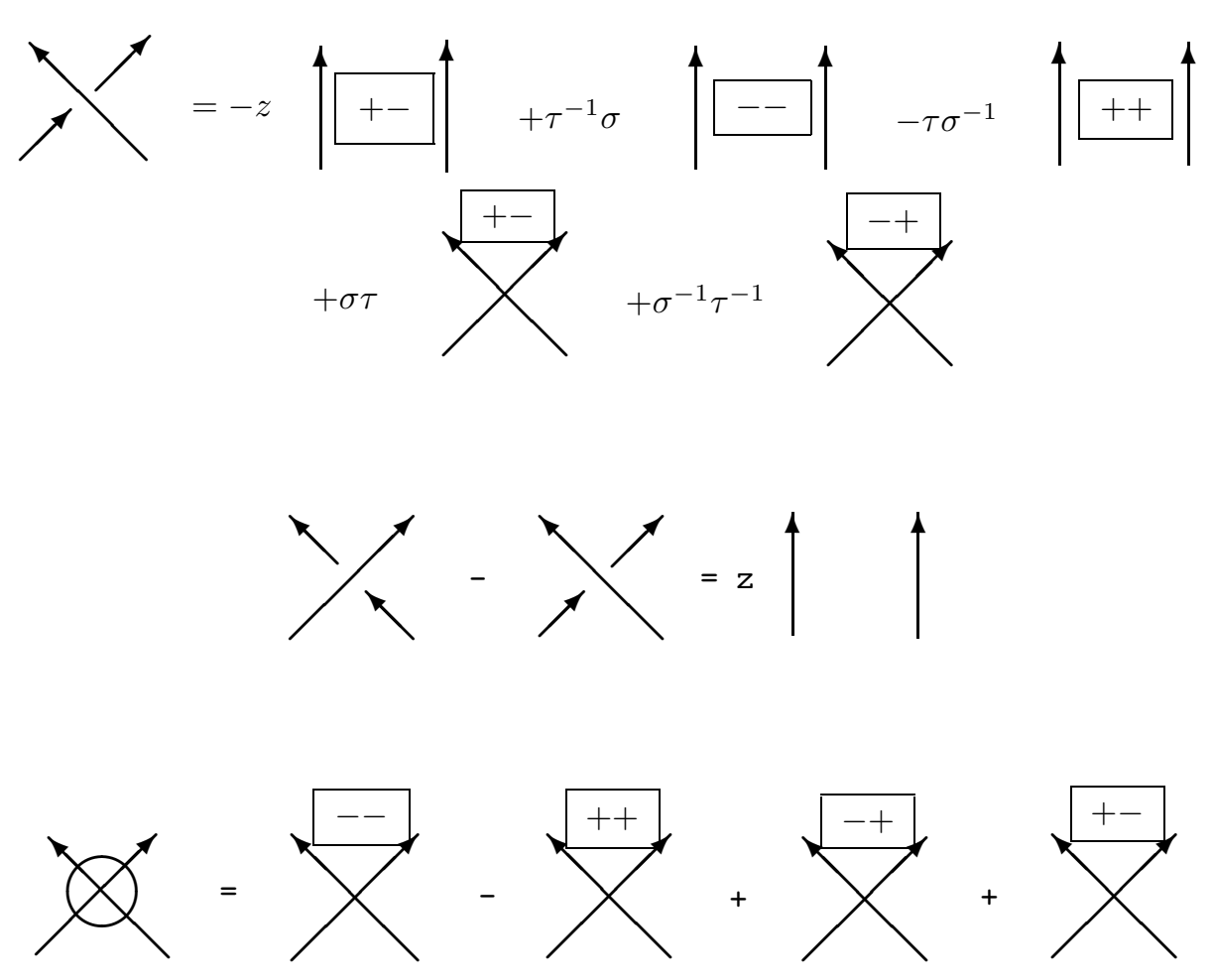

Figure 12 - Expansion Formulas for the State Summation

Remark. It should be remarked that there is a natural multivariable version of the polynomial invariant $G_{K}(s, t)$ to a polynomial invariant $G_{K}\left(s, t_{1}, \ldots, t_{\mu}\right)$ where $\mu$ denotes the number of components in the link $K$. Each link component receives a separate variable, and the biquandle relations at a crossing are determined by the label for the undercrossing segment at that crossing. The procedure of extending through the exterior algebra still goes through to produce a quantum model for this many-variable polynomial. We will study this generalized invariant in a separate paper. 

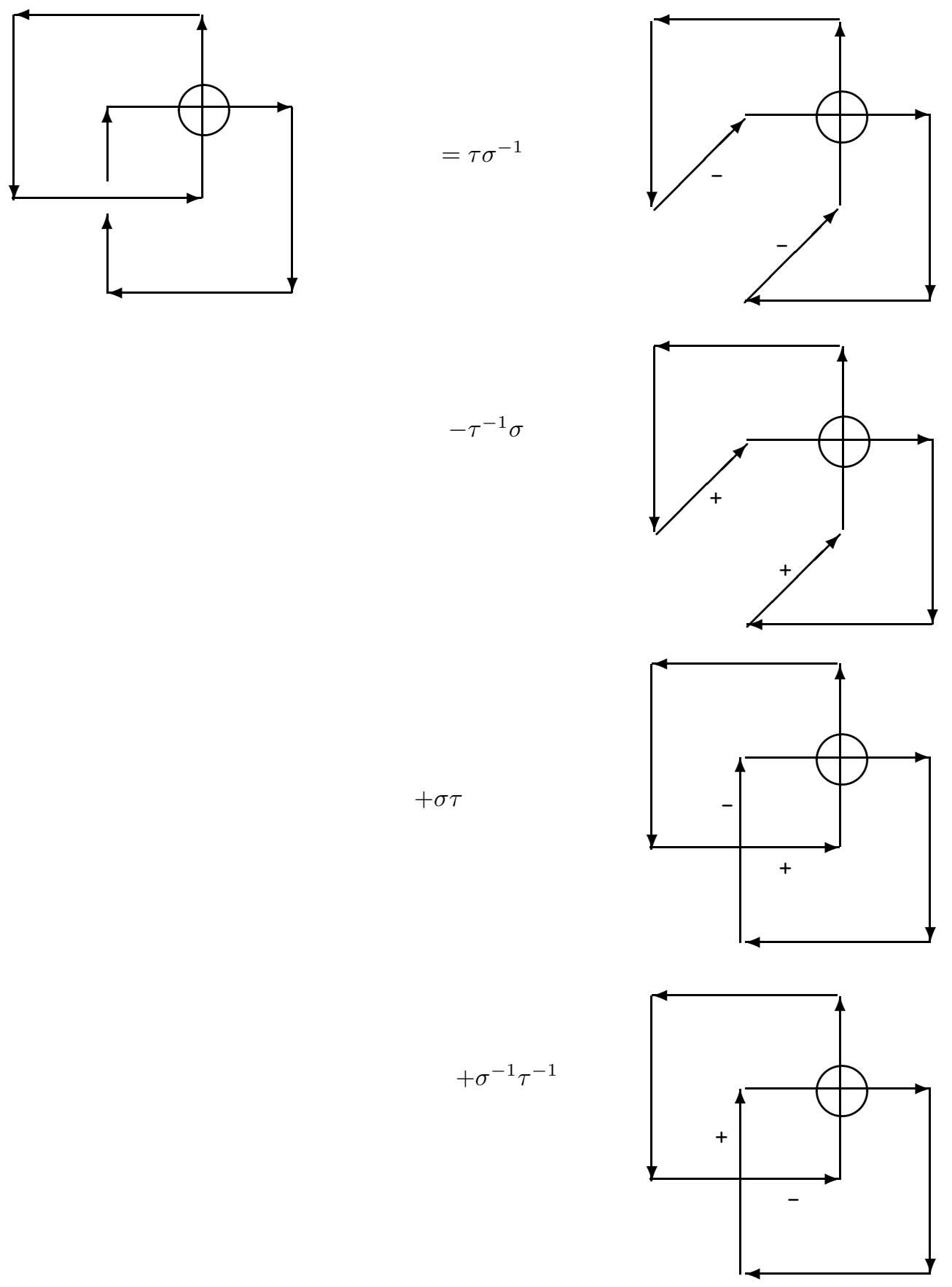

Figure 13 - Expansion Formulas for Virtual Hopf Link 
BI-ORIENTED QUANTUM ALGEBRAS, AND A GENERALIZED ALEXANDER POLYNOMIAL FOR VIRTUAL LINIRS

\section{Bi-oriented Quantum Algebras}

It is the purpose of this section to place our work with the generalized Alexander polynonmial in a context of bi-oriented quantum algebras. To do this (and to define the concept of a bi-oriented quantum algebra) we need to first recall the notion of an oriented quantum algebra. An oriented quantum algebra $(A, \rho, D, U)$ is an abstract model for an oriented quantum invariant of classical links [6], [7]. This model is based on a solution to the Yang-Baxter equation and some extra structure that serves to make an invariant possible to construct. The definition of an oriented quantum algebra is as follows: We are given an algebra $A$ over a base ring $k$, an invertible solution $\rho$ in $A \otimes A$ of the Yang-Baxter equation (in the algebra formulation of this equation - see the Remark below), and commuting automorphisms $U, D: A \longrightarrow A$ of the algebra, such that

$$
\begin{gathered}
(U \otimes U) \rho=\rho, \\
(D \otimes D) \rho=\rho, \\
\left.\left[\left(1_{A} \otimes U\right) \rho\right)\right]\left[\left(D \otimes 1_{A^{o p}}\right) \rho^{-1}\right]=1_{A \otimes A^{o p}},
\end{gathered}
$$

and

$$
\left.\left[\left(D \otimes 1_{A^{o p}}\right) \rho^{-1}\right]\left[\left(1_{A} \otimes U\right) \rho\right)\right]=1_{A \otimes A^{\circ p}} .
$$

The last two equations say that $\left.\left[\left(1_{A} \otimes U\right) \rho\right)\right]$ and $\left[\left(D \otimes 1_{A^{\text {op }}}\right) \rho^{-1}\right]$ are inverses in the algebra $A \otimes A^{o p}$ where $A^{o p}$ denotes the opposite algebra.

When $U=D=T$, then $A$ is said to be balanced. In this case

$$
\begin{gathered}
(T \otimes T) \rho=\rho \\
\left.\left[\left(1_{A} \otimes T\right) \rho\right)\right]\left[\left(T \otimes 1_{A^{o p}}\right) \rho^{-1}\right]=1_{A \otimes A^{o p}}
\end{gathered}
$$

and

$$
\left.\left[\left(T \otimes 1_{A^{o p}}\right) \rho^{-1}\right]\left[\left(1_{A} \otimes T\right) \rho\right)\right]=1_{A \otimes A^{o p}} .
$$

In the case where $D$ is the identity mapping, we call the oriented quantum algebra standard. As we saw in [7], the invariants defined by Reshetikhin and Turaev (associated with a quasi-triangular Hopf algebra) arise from standard oriented quantum algebras. It is an interesting structural feature of algebras that we have elsewhere [4] called quantum algebras (generalizations of quasi-triangular Hopf algebras) that they give rise to standard oriented quantum algebras. Note that the term quantum algebra as used here is more specific than the $Q A$ (quantum algebra) designation that is used in archived papers related to Hopf algebras and quantum groups.

Appropriate matrix representations of oriented quantum algebras or the existence of certain traces on these algebras allow the construction of oriented invariants of knots and links. These invariants include all the known quantum link invariants at the time of this writing. 
Remark. Note that we have the Yang-Baxter elements $\rho$ and $\rho^{-1}$ in $A \otimes A$. We assume that $\rho$ and $\rho^{-1}$ satisfy the algebraic Yang-Baxter equation. This equation (for $\rho$ ) states

$$
\rho_{12} \rho_{13} \rho_{23}=\rho_{23} \rho_{13} \rho_{12}
$$

where $\rho_{i j}$ denotes the placement of the tensor factors of $\rho$ in the $i$-th and $j$-th tensor factors of the triple tensor product $A \otimes A \otimes A$ with the unit element of $A$ placed in the remaining factor.

We write $\rho=\Sigma e \otimes e^{\prime}$ and $\rho^{-1}=\Sigma E \otimes E^{\prime}$ to indicate that these elements are sums of tensor products of elements of $A$. The expression $e \otimes e^{\prime}$ is thus a generic element of the tensor product. However, we often abbreviate and write $\rho=e \otimes e^{\prime}$ and $\rho^{-1}=E \otimes E^{\prime}$ where the summation is implicit. We refer to $e$ and $e^{\prime}$ as the signifiers of $\rho$, and $E$ and $E^{\prime}$ as the signifiers of $\rho^{-1}$. For example, $\rho_{13}=e \otimes 1 \otimes e^{\prime}$ in $A \otimes A \otimes A$.

Braiding operators, as they appear in knot theory, differ from the algebraic YangBaxter elements by a permutation of tensor factors. This point is crucial to the relationship of oriented quantum algebras and invariants of knots and links.

We extend the concept of oriented quantum algebra by adding a second solution to the Yang-Baxter equation $\gamma$ that will take the role of the virtual crossing.

Definition. A bi-oriented quantum algebra is a quintuple $(A, \rho, \gamma, D, U)$ such that $(A, \rho, D, U)$ and $(A, \gamma, D, U)$ are oriented quantum algebras and $\gamma$ satisfies the following properties:

1. $\gamma_{12} \gamma_{21}=1_{A \otimes A}$. (This is the equivalent to the statement that the braiding operator corresponding to $\gamma$ is its own inverse.)

2. The following mixed identities involving $\rho$ and $\gamma$ are satisfied. These correspond to the braiding versions of the virtual detour move of type three that involves two virtual crossings and one real crossing.

$$
\begin{gathered}
\gamma_{12} \rho_{13} \gamma_{23}=\gamma_{23} \rho_{13} \gamma_{12} \\
\gamma_{12} \gamma_{13} \rho_{23}=\rho_{23} \gamma_{13} \gamma_{12} \\
\rho_{12} \gamma_{13} \gamma_{23}=\gamma_{23} \gamma_{13} \rho_{12} .
\end{gathered}
$$

By extending the methods of [7], it is not hard to see that a bi-oriented quantum algebra will always give rise to invariants of virtual links up to the type one moves (framing and virtual framing).

Any oriented quantum algebra $(A, \rho, D, U)$ gives rise to a bi-oriented quantum algebra by taking $\gamma$ to be the identity element in $A \otimes A$. This corresponds to associating a simple permutation (transposition) to the virtual crossing. The resulting invariants of virtuals are worth investigating. In [9] the corresponding generalization of the Jones polynomial is studied. The bi-oriented quantum algebras form a context for these invariants. 
In the case of the generalized Alexander polynomial studied in this paper, the matrix model and state model for $Z(K)$ translate directly into a specific example of a bi-oriented balanced quantum algebra $(A, \rho, \gamma, T)$ (It is a balanced bi-oriented quantum algebra.) with the underlying algebra $A$ the algebra of elementary matrices as in [7]. In making this translation to the algebra, one must take the matrices of the matrix model and compose with a permutation. In our case the matrix $\gamma$ is a diagonal $4 \times 4$ matrix with three ones and one minus one in that order on the diagonal. This is the matrix obtained from the matrix $\eta$ that we used for the virtual crossing.

$$
\gamma=\left[\begin{array}{cccc}
1 & 0 & 0 & 0 \\
0 & 1 & 0 & 0 \\
0 & 0 & 1 & 0 \\
0 & 0 & 0 & -1
\end{array}\right] .
$$

The matrix $\rho$ is obtained from the braiding matrix $R$ by permuting the two middle columns (because $\rho_{c d}^{a b}=R_{c d}^{b a}$ ).

$$
\rho=\left[\begin{array}{cccc}
\sigma^{-1} \tau & 0 & 0 & 0 \\
0 & \sigma \tau & z & 0 \\
0 & 0 & \sigma^{-1} \tau^{-1} & 0 \\
0 & 0 & 0 & -\sigma \tau^{-1}
\end{array}\right] .
$$

On elementary matrices $E_{b}^{a}$ the transformation $T$ is given by the formula $T\left(E_{b}^{a}\right)=$ $i^{b-a}$ where $i^{2}=-1$. (See Section 4.2 of [7].) This completes the description of the bi-oriented quantum algebra that corresponds to the generalized Alexander polynomial of this paper.

The main algebraic point about the bi-oriented quantum algebra for the generalized Alexander polynomial is that the operator $\gamma$ for the virtual crossing is not the identity operator, and that this non-triviality is crucial to the structure of the invariant. We will investigate bi-oriented quantum algebras and other examples of virtual invariants derived from them in a subsequent paper.

\section{References}

[1] R. Fenn, M. Jordan and L. H. Kauffman, Biracks, Biquandles and Virtual Knots, in preparation.

[2] F. Jaeger, L. H. Kauffman and H. Saleur, The Conway polynomial in $R^{3}$ and in thickened surfaces: A new determinant formulation, J. Comb. Theory Ser. B Vol. 61 (1994), 237-259.

[3] L. H. Kauffman and H. Saleur, Free fermions and the Alexander-Conway polynomial, Comm. Math. Phys. 141, 293-327 (1991).

[4] Louis H. Kauffman, Gauss Codes, quantum groups and ribbon Hopf algebras, Reviews in Mathematical Physics 5 (1993), 735-773. (Reprinted in [5], 551-596.)

[5] Louis H. Kauffman, "Knots and Physics," World Scientific, Singapore/New Jersey/London/Hong Kong, 1991, 1994.

[6] Louis H. Kauffman and David E. Radford, Oriented quantum algebras and invariants of knots and links. J. Alg. Vol. 246 (2001), 253-291.

[7] Louis H. Kauffman and David E. Radford, Oriented quantum algebras, categories and invariants of knots and links. JKTR Vol. 10, No. 7 (2001), 1047-1084.

[8] Scott Carter and Dan Silver (private conversation).

[9] Louis H. Kauffman, Virtual Knot Theory , European J. Comb. (1999) Vol. 20, 663-690. 
[10] Louis H. Kauffman, A Survey of Virtual Knot Theory in Proceedings of Knots in Hellas '98, World Sci. Pub. 2000 , pp. 143-202.

[11] Louis H. Kauffman, Detecting Virtual Knots, to appear in Atti. Sem. Mat. Fis., Univ. Modena (2001).

[12] Louis H. Kauffman, Diagrammatic Knot Theory (in preparation).

[13] Toshimasa Kishino and Shin Satoh, A note on non-classical virtual knots, (to appear in JKTR).

[14] J. Sawollek, On Alexander-Conway polynomials for virtual knots and links, arXiv:math.GT/9912173 21Dec 1999.

[15] D. S. Silver and S. G. Williams, Alexander Groups and Virtual Links, JKTR Vol 10 (2001) 151-160.

[16] J.S.Carter and M. Saito, Diagrammatic invariants of knotted curves and surfaces, (unpublished manuscript - 1992).

Department of Mathematics, Statistics and Computer Science, University of Illinois at Chicago, 851 South Morgan St., Chicago IL 60607-7045, U.S.A.

E-mail address: kauffman@uic.edu

Department of Mathematics, Statistics and Computer Science, University of Illinois at Chicago, 851 South Morgan St., Chicago IL 60607-7045, U.S.A.

E-mail address: radford@math.uic.edu 